\title{
Nasopharyngeal carcinoma among the pediatric patients in a non-endemic region: our experience at a tertiary care teaching hospital in Eastern India
}

\author{
Santosh Kumar Swain ${ }^{1 *}$, Smrutipragnya Samal ${ }^{2}$, Jatindra Nath Mohanty ${ }^{2}$ and Jasahree Choudhury ${ }^{3}$
}

\begin{abstract}
Background: Nasopharyngeal carcinoma (NPC) is an extremely rare malignant lesion among the pediatric age group. The relative rarity of pediatric NPC makes the diagnosis difficult. This rarity is often associated with delayed diagnosis which may lead to advanced loco-regional disease. Here, we study the clinical presentations, investigations, and treatment of nasopharyngeal carcinoma in the pediatric age group in a non-endemic region.

Result: This is a retrospective study where 21 pediatric patients were enrolled with age under 18 years. They were managed at a tertiary care teaching hospital between December 2010 and January 2019. Majority of the patients in this study were boys (66.7\%). All children diagnosed with NPC were treated with radiotherapy covering entire nasopharynx and some children with chemotherapy. Until the patient is in a late stage, most children diagnosed with NPC were presenting with symptoms of neck mass (90.5\%), bleeding from the nose (66.7\%), nasal blockage (57.1\%), and hearing loss (47.6\%). Pathological report revealed WHO type III in the majority of the patients. All patients were treated with radiotherapy to primary and enlarged neck nodes.

Conclusion: Children with NPC have excellent survival except for those with distant metastatic disease. NPC in the pediatric age is usually not suspected clinically until patient in late stage. The TNM staging has the most relevant prognostic factor. Unfortunately, NPC tends to be locally advanced at the time of diagnosis in the pediatric age group and is sometimes associated with distant metastasis. In our study, most children were diagnosed with NPC along with neck node enlargement and were treated with radiotherapy. The diagnosis of pediatric NPC should prompt timely treatment.
\end{abstract}

Keywords: Pediatric age, Nasopharyngeal carcinoma, Chemoradiation

\section{Background}

Nasopharyngeal carcinoma (NPC) is a malignant neoplasm arising from the epithelial cells lining the nasopharynx. NPC is a head and neck malignancy with remarkable ethnic and geographical variations in incidence. It is a rare malignancy with an incidence in high-

\footnotetext{
* Correspondence: santoshvoltaire@yahoo.co.in

'Department of Otorhinolaryngology, IMS and SUM Hospital, Siksha "O" Anusandhan University (Deemed to be), K8, Kalinganagar, Bhubaneswar, Odisha 751003 , India

Full list of author information is available at the end of the article
}

risk areas as 20 per 100,000 persons in a year and lower rates are seen in the pediatric age [1]. NPC is a rare tumor in children younger than 10 years of age, and the incidence rises gradually with the age of the patient. The annual incidence of NPC is around 0.5 per million children (10-14 years of age) and 1.1 million adolescents (15 to 17 years of age) [2]. The incidence of childhood NPC is less than $1 \%$ of all pediatric malignant neoplasms, as this head and neck tumor is predominantly seen in adults [3]. The high-risk regions for nasopharyngeal carcinoma are Southern China, Taiwan, and Hong Kong 
[3]. NPC is extremely rare among children regardless of the different regions in the world. Approximately, $5 \%$ of the primary malignant neoplasms in children arise from the head and neck area [4], whereas the NPC represents around $2 \%$ of the head and neck malignant neoplasm in the pediatric age group [1]. As this is rare in the pediatric age group and its non-specific clinical presentation like nasal congestion, lymphadenopathy, and ear symptoms, the diagnosis is often delayed because of initial thinking of benign etiologies. The standard treatment of NPC is radiotherapy. In this study, we discuss the clinical profile, management, and outcome of pediatric NPC at a tertiary care teaching hospital in Eastern India.

\section{Methods}

This study was conducted at the Otolaryngology and Pediatric Departments of a tertiary care teaching hospital in the eastern part of India basing on the data of children and adolescent patients diagnosed with nasopharyngeal carcinoma. The only inclusion criteria were patients younger than 18 years with NPC between December 2010 to January 2019 (Table 1). This retrospective study was approved by the institutional ethical committee of our medical college. Written consents were taken from the parents of the children participating in this study. All children of NPC underwent a detailed history taking, a full head and neck examination, diagnostic nasal endoscopy, and audiological examinations. Computed tomography (CT) scan of the neck and nasopharynx was done routinely in all diagnosed cases of NPC. All children diagnosed with NPC were treated with radiotherapy covering entire nasopharynx and some children with chemotherapy. All children of NPC were followed regularly with physical examination and fiberoptic nasopharyngolaryngoscopy every 3 months for the first 2 years, every 6 months for the next 5 years, and then yearly thereafter. Hormonal tests like thyroid function tests, growth hormone, follicle-stimulating hormone (FSH), and luteinizing hormone (LH) were done for the majority of the patients after chemoradiation.

\section{Results}

Twenty-one children aged between 6 to 18 years diagnosed with nasopharyngeal carcinoma participated in this study. There were 14 boys (66.7\%) and 7 girls (33.3\%) in this study. The median age at the diagnosis of

Table 1 Diagnosed cases of NPC during study period

\begin{tabular}{ll}
\hline Years & Number of patients diagnosed \\
\hline $2010-2013$ & 9 \\
$2014-2016$ & 8 \\
$2017-2019$ & 4 \\
\hline
\end{tabular}

NPC was 14 years (range 6-18 years). The most common clinical presentation was neck swelling (Fig. 1) in 19 (90.5\%) patients, followed by nasal blockage, bleeding from the nose, and hearing impairment in 14 (66.7\%), 14 $(66.66 \%)$, and $12(57.1 \%)$ of cases respectively (Table 2$)$. As per histopathological report of our patients, six patients had WHO type I (28.6\%), four patients had WHO type II (19\%), and eleven patients had WHO type III nasopharyngeal carcinoma (52.4\%) (Fig. 2) (Table 3). The site for a primary lesion at nasopharynx was as follows: the lateral wall (Fig. 3 ) in 9 cases (42.8\%), posterosuperior wall in 8 cases (38\%), and the entire nasopharynx in 4 cases (19\%) (Table 3). Four cases (19.04\%) showed skull base invasion. Neck node enlargement was seen in 19 cases (90.5\%). N staging was as follows: $\mathrm{N} 1$ in 14 cases $(66.7 \%), \mathrm{N} 2$ in 5 cases $(23.8 \%)$, and N3 in 2 cases (9.5\%) (Table 4). All the children with NPC were treated with radiotherapy with an overall good prognosis. Radiation of $1.8 \mathrm{~Gy} /$ day for 5 days a week and a total dose of $40-44 \mathrm{~Gy}$ is given to the primary site. Seven children (5 cases of N2 and 2 cases of N3) were administered with 2-3 cycles of cisplatin-based chemotherapy (cisplatin $80 \pm 100 \mathrm{mg} / \mathrm{m}^{2}, 5$-flurorouracil $600 \mathrm{mg} / \mathrm{m}^{2}$, bleomycin $15 \mathrm{mg} / \mathrm{m}^{2}$, epirubicin $100 \mathrm{mg} / \mathrm{m}^{2}$, methotrexate $40 \mathrm{mg} / \mathrm{m}^{2}$ ) after completion with radiotherapy and/or chemotherapy (if distant metastasis were present). So out of 21 patients, 7 patients were given adjuvant chemotherapy. NPC with neck node metastasis/

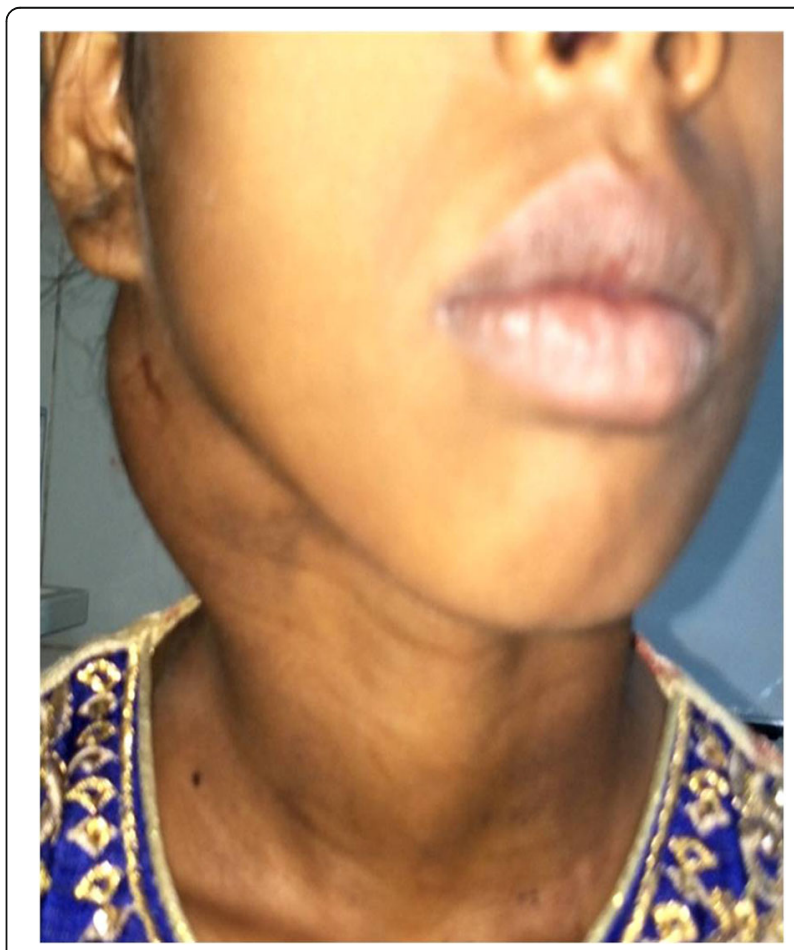

Fig. 1 A female child with nasopharyngeal carcinoma presenting with right side neck mass 
Table 2 Clinical profile of pediatric nasopharyngeal carcinoma

\begin{tabular}{|c|c|c|c|c|c|c|}
\hline Cases & $\begin{array}{l}\text { Age } \\
\text { (years) }\end{array}$ & Gender & $\begin{array}{l}\text { Neck node swelling (yes/ } \\
\text { no) }\end{array}$ & $\begin{array}{l}\text { Nasal bleeding (yes/ } \\
\text { no) }\end{array}$ & $\begin{array}{l}\text { Nasal block (yes/ } \\
\text { no) }\end{array}$ & $\begin{array}{l}\text { Aural symptoms (otalgia/hearing loss) (yes/ } \\
\text { no) }\end{array}$ \\
\hline 1 & 6 & $M$ & $Y$ & $Y$ & $Y$ & $Y$ \\
\hline 2 & 7 & $M$ & $Y$ & $Y$ & $N$ & $Y$ \\
\hline 3 & 7 & $M$ & Y & $\mathrm{N}$ & $\mathrm{N}$ & $N$ \\
\hline 4 & 11 & $\mathrm{~F}$ & $Y$ & $N$ & $Y$ & $N$ \\
\hline 5 & 12 & $M$ & $Y$ & $N$ & Y & Y \\
\hline 6 & 12 & $\mathrm{~F}$ & $Y$ & Y & $\mathrm{N}$ & Y \\
\hline 7 & 13 & $\mathrm{~F}$ & $Y$ & Y & $Y$ & $N$ \\
\hline 8 & 13 & M & Y & Y & $Y$ & Y \\
\hline 9 & 13 & $M$ & $Y$ & Y & $N$ & Y \\
\hline 10 & 14 & $M$ & $Y$ & $Y$ & $Y$ & $N$ \\
\hline 11 & 14 & $\mathrm{~F}$ & $\mathrm{~N}$ & Y & $N$ & $N$ \\
\hline 12 & 14 & $M$ & $Y$ & $Y$ & $Y$ & $N$ \\
\hline 13 & 14 & $M$ & Y & $N$ & $N$ & Y \\
\hline 14 & 14 & $\mathrm{~F}$ & $Y$ & $\mathrm{~N}$ & $Y$ & Y \\
\hline 15 & 15 & $\mathrm{~F}$ & $Y$ & Y & $Y$ & $N$ \\
\hline 16 & 15 & $\mathrm{~F}$ & $Y$ & $\mathrm{~N}$ & $Y$ & $Y$ \\
\hline 17 & 16 & M & $Y$ & Y & $N$ & $Y$ \\
\hline 18 & 16 & $M$ & $Y$ & $Y$ & $Y$ & $N$ \\
\hline 19 & 17 & $M$ & $Y$ & $N$ & Y & Y \\
\hline 20 & 18 & $M$ & $N$ & Y & $N$ & $N$ \\
\hline 21 & 18 & $M$ & Y & $Y$ & $N$ & $N$ \\
\hline
\end{tabular}

enlargement showed an incremental decrease in the size after treatment.

There was no involvement of any cranial nerve in the cases of this study. After completion of treatment with radiotherapy and/or chemotherapy (if distant metastasis

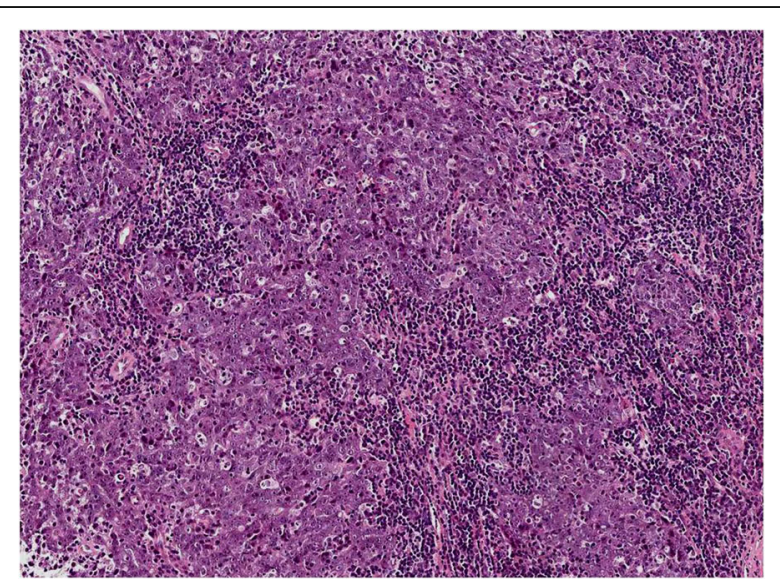

Fig. 2 Histopathology picture of nasopharyngeal carcinoma showing invasive cords and nests of oval to cuboidal cells with large hyperchromatic and mitotically active nuclei were present), one patient was lost to follow up for unknown reasons after treatment without any information to treating doctor. One patient quits his therapy during middle of treatment.

Three children (two of them with T2N2M0 and one with T2N3M0) presented with a recurrence at the primary site after 10 months of treatment and 4th case of recurrent lesions (T2N2M0) identified after 18 months of treatment. All 4 cases (19.04\%) of recurrent lesions were treated with surgery, re-irradiation, and adjuvant chemotherapy. Two patients died due to distant metastasis and recurrence at primary sites. Seventeen cases showed necrotic changes in enlarged lymph nodes (80.9\%) in contrast CT scan of the neck. Complete remission was reported in $17 / 21$ cases $(80.9 \%)$. The mortality was reported only in 2 cases $(9.52 \%)$ and 2 patients (9.5\%) lost follow up.

Table 3 Pathology/WHO classification

\begin{tabular}{lll}
\hline WHO & Number of patients & $\%$ \\
\hline WHO I & 6 & 28.6 \\
WHO II & 4 & 19 \\
WHO III & 11 & 52.4 \\
\hline
\end{tabular}




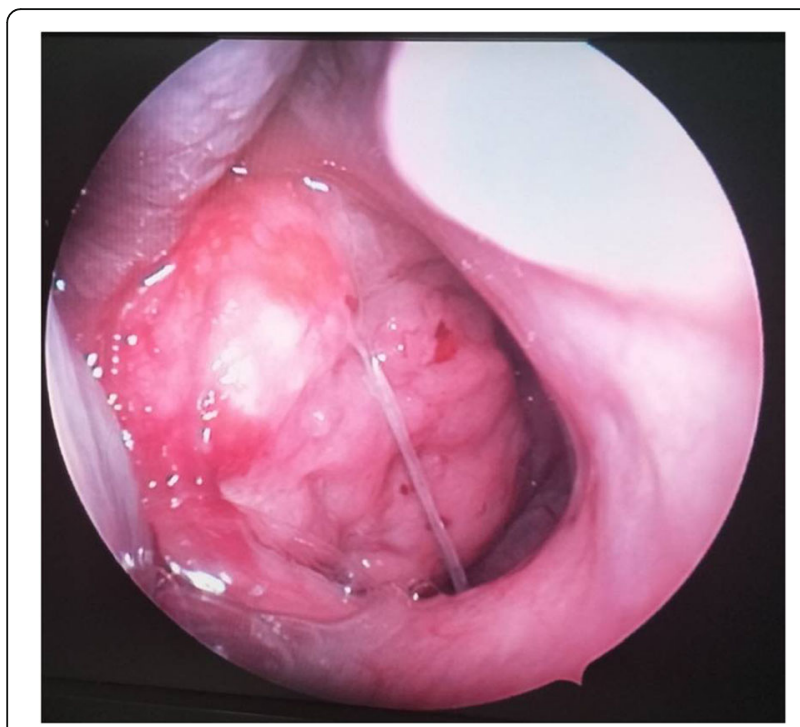

Fig. 3 Endoscopic picture showing an irregular mass at the right posterosuperior part of the nasopharynx

\section{Discussion}

Nasopharyngeal carcinoma is a rare malignant tumor seen in the head and neck region. The causes are multifactorial and include viral, environmental, and genetic [5]. NPC is a head and neck malignancy commonly seen in southern China, south east Asia, and North Africa [5]. Historically, NPC has been thought as a disease of elderly people as the incidence of NPC increases with age and attends peak at 50 to 59 years of age with a small peak in late childhood [3]. The incidence is higher among males than females across the world, particularly in endemic area [4]. Similar higher incidences among males are seen in this study. Pediatric NPC is a rare

Table 4 Imaging in pediatric NPC

\begin{tabular}{lll}
\hline CT Findings $(n=21)$ & Number of children & $\%$ \\
\hline Site of lesion & 9 & 42.9 \\
Lateral wall & 8 & 38.1 \\
Posterosuperior wall & 4 & 19 \\
Entire nasopharynx & 4 & 19 \\
Skull base invasion & & \\
Neck node metastasis & 14 & 66.7 \\
N1 & 5 & 23.8 \\
N2 & 2 & 9.5 \\
N3 & 17 & 80.9 \\
Necrotic changes in the neck nodes & & \\
Distant metastasis & 20 & 95.2 \\
M0 & 1 & 4.8 \\
M1
\end{tabular}

neoplasm, and the incidence varies from endemic to non-endemic areas. In the present study, the incidence among the boys and girls are in the ratio of 2.2:1, consistent with previous studies [6-8].

NPC is pathologically classified (WHO classification) into three categories: keratinizing squamous cell carcinoma (WHO type I), non-keratinizing squamous cell carcinoma (WHO type II), and undifferentiated carcinoma (WHO type III) (Table 5) [9]. Majority of the cases are undifferentiated carcinoma (WHO type III) in advanced stage [10]. NPC is usually caused due to genetic susceptibility, environmental factors like exposure to chemical carcinogens, and infections with Epstein-Barr virus. Raised antibody titers to Epstein-Barr virus antigens may be used as markers [11].

The clinical presentations of the pediatric NPC are nasal bleeding, nasal blockage, neck swelling, and ear symptoms like deafness, tinnitus, and headache. Commonest site of origin for NPC is fossa of Rosenmuller. This is an occult location and the patient is usually asymptomatic for a prolonged period. The classical clinical presentations such as neck mass, epistaxis, nasal obstruction, and serous otitis media occur infrequently. Bone erosion at the skull base region with or without affecting cranial nerves is common at the time of diagnosis. Cranial nerves III to VI are commonly involved in NPC. In contrast to other cancers of the head and neck, NPC has a greater tendency for early metastasis [12]. As there is rich lymphatic drainage of the nasopharynx, bilateral neck node metastasis is often the first clinical sign. The rarity of NPC in children often leads to delayed diagnosis, so childhood NPC is associated with advanced loco-regional spread and higher chance of distant metastasis [13]. In many cases, the diagnosis is delayed as the cervical lymphadenopathy suspected to be of infectious cause. Unilateral serous otitis media is a common presentation in adult patient with NPC, whereas in children this is not an atypical presentation. Otalgia and cervical lymphadenopathy are in favor of infectious etiology in the pediatric age group. In the absence of improvement with the course of antibiotic treatment, further workup is required. Persistent unilateral otological findings need flexible nasopharyngolaryngoscopic examination to rule out nasopharyngeal

Table 5 WHO classification (pathology) for NPC

\begin{tabular}{ll}
\hline Types & Cellular characteristics \\
\hline Type I & Keratinizing squamous cell carcinoma \\
Type II & Non-keratinizing (transitional) carcinoma \\
& - Without lymphoid stroma (intermediate cell) \\
& - With lymphoid stroma (lympho-epithelial) \\
Type III & Undifferentiated (anaplastic) carcinoma \\
& - Without lymphoid stroma (clear cell) \\
& With lymphoid stroma (lympho-epithelial) \\
\hline
\end{tabular}


pathology. The peak incidence of NPC in children of non-endemic region is in the age of 15 to 19 years with male to female ratio of $>2: 1$ [14]. As there is no exact time-frame for initiating a malignancy work up in the pediatric age group with one-sided otological symptoms, a higher degree of suspicion may be needed in childhood.

There are certain characteristics specific to pediatric NPC that are markedly different from the adult population: being pediatric NPC is often classified as undifferentiated carcinoma, advanced loco-regional disease in the first diagnosis, higher prevalence of distant metastasis and closely associated with Epstein-Barr virus (EBV) etiology, and higher prevalence of treatment/therapy-related complications in comparison to the adult group [6, 15]. MRI is usually helpful to provide accurate imaging for detecting perineural and intracranial spread and also accurate in detecting the marrow infiltration whereas cortical destruction of bone is better assessed by CT scan [16]. Both imaging are almost of the same accuracy for assessing the lymph nodes. Because of the limited availability of pediatric MRI in our center, all of our patients were evaluated by CT scan. Diagnostic nasal endoscopy with biopsy is a must for confirmation of the diagnosis.

Radiotherapy is the treatment of choice, and the neoadjuvant chemotherapy is given in advanced cases. Radiation of $50 \mathrm{~Gy}$ is often desirable for neck nodes. The chemotherapy includes methotrexate, bleomycin, and cisplatin [17]. Nasopharyngeal carcinomas are more radiosensitive [18]. Our patients showed significant improvement after radiotherapy and chemotherapy and now under follow up. The side effects of radiotherapy to the head and neck area vary, most often patients complain xerostomia. The chemotherapeutic agent such as cisplatin is commonly prescribed and leads to ototoxicity, typically bilateral and irreversible [19]. Loss of hearing in childhood can affect speech, cognitive, and social development. The future treatment needs avoidance of such drugs. NPC is usually inoperable due to the complex anatomical location of disease and surgery of this primary neoplasm is confined to biopsy only. The radical neck dissection is considered if the primary tumor seems to be controlled or there are persistent neck nodes or recurrence in the nodes after chemoradiation or radiation alone [20]. Patel et al. published 5-year survival of pediatric patients under the age of 18 years and adult patients, $77 \pm 7.3 \%$ and $83 \pm 3.9 \%$ respectively [21]. The risk of developing second cancer after treatment is significantly higher in pediatric patients with NPC [7]. In children, early diagnosis of NPC before loco-regional spread will hopefully avoid the extent of radiation-associated comorbidities. Radiation dose should be reduced for patients with NPC with positive outcome to neo-adjuvant chemotherapy. Intensitymodulated radiotherapy (IMRT) is a breakthrough in the treatment of NPC which provides highly conformal dose distributions to the targeted site with simultaneously avoiding radiation to normal tissue which helps to improve survival and decrease late toxicity [22]. The overall outcome is almost similar between adult and pediatric patients with 5-year disease-specific survival in the range between 38 and 51\% [23]. However, this outcome contradicts to Sultan et al. [13], who documents that patients of NPC with ages between 20 and 45 years old have almost double risk for mortality in comparison to child or adolescent. But the children or adolescents have a higher risk for getting second cancer compared to adults, which may be due to treatment-related complications [13]. Neo-adjuvant chemotherapy followed by concurrent chemo-radiotherapy has event-free survival rates of around $70-80 \%$ for pediatric age groups with NPC [24]. However, outcomes for pediatric patients with relapsed NPC remain poor [25]. Most of the patients with relapsed NPC present with distant metastatic disease [26]. Alone radiotherapy is often inadequate for treating relapsed NPC as most of the relapse occurs at the distant sites. Furthermore, the radiotherapy doses required to treat distant metastatic NPC may lead to severe organ toxicity. So, chemotherapy is usually needed to treat the majority of pediatric patients. Early diagnosis and treatment of pediatric NPC helps to prevent morbidity and mortality.

\section{Conclusion}

Nasopharyngeal carcinoma in the pediatric age group is a very rare clinical entity. It presents with advanced loco-regional disease if diagnosed in a late stage. Early administration of treatment is required for childhood NPC. The treatment of pediatric NPC is essentially multimodal and includes radiotherapy and chemotherapy. High dose of radiation has an excellent control of the NPC at the cost of toxicity with serious consequences in the pediatric age group. Reduction of radiation dose to the nasopharynx with the help of neoadjuvant or concomitant chemotherapy helps to reduce late toxicity along with local control of the tumor. NPC should be considered as a differential diagnosis when child presents with neck swelling, nasal obstruction, nasal bleeding, unilateral conductive hearing loss due to middle ear effusion, and growth in the nasopharynx.

\section{Abbreviations}

NPC: Nasopharyngeal carcinoma; EBV: Epstein-Barr virus; IMRT: Intensitymodulated radiotherapy; CT Scan: Computed tomography scan; WHO: World Health Organization; FSH: Follicle-stimulating hormone; LH: Luteinizing hormone; Chemoradiation: Treatment which combines chemotherapy with radiation therapy 


\section{Acknowledgements}

The authors are thankful to Prof. Manoj Ranjan Nayak, Founder president, Siksha "O" Anusandhan University, Bhubaneswar, India, for his guidance and support.

\section{Authors' contributions}

SKS conceived and supervised the study. SS and JNM collected samples and had also done data collection. SKS, SS, JNM, and JC interpreted data and prepared the manuscript. All authors read and approved the final manuscript.

\section{Funding}

Nil

\section{Availability of data and materials}

Data and material were taken from IMS \& SUM Hospital, Siksha "O"

Anusandhan University, Bhubaneswar, India.

\section{Ethics approval and consent to participate}

Written consent of parent of each participant child was obtained for this study. Name of the ethics committee: Institutional Ethics Committee-Institute of Medical Sciences (IMS) and SUM Hospital, Siksha "O" Anusandhan University, Bhubanenswar, India. Committee Reference number: Ref.No/DMR/ IMS.SH/SOA/160203.

\section{Consent for publication}

Parental consent had been obtained for publication.

\section{Competing interests}

$\mathrm{Nil}$

\section{Author details}

'Department of Otorhinolaryngology, IMS and SUM Hospital, Siksha "O" Anusandhan University (Deemed to be), K8, Kalinganagar, Bhubaneswar, Odisha 751003, India. 'Medical Research Laboratory, IMS and SUM Hospital, Siksha "O" Anusandhan University (Deemed to be), K8, Kalinganagar, Bhubaneswar, Odisha 751003, India. ${ }^{3}$ Department of Pediatrics, IMS and SUM Hospital, Siksha "O" Anusandhan University (Deemed to be), K8, Kalinganagar, Bhubaneswar, Odisha 751003, India.

Received: 10 June 2019 Accepted: 6 July 2020

Published online: 01 August 2020

\section{References}

1. Sun LM, Li Cl, Huang EY, Vaughan TL (2007) Survival differences by race in nasopharyngeal carcinoma. Am J Epidemiol. 165:271-278

2. Pastore G, De Salvo GL, Bisogno G et al (2009) Evaluating the access to pediatric cancer care centers of children and adolescents with rare tumors in Italy: the TREP project. Pediatr Blood Cancer. 53:152-155

3. Chang ET, Adami HO (2006) The enigmatic epidemiology of nasopharyngeal carcinoma. Cancer Epidemiol Biomarkers Prev 15:1765-1777

4. Kao WC, Chen JS, Yen CJ (2016) Advanced nasopharyngeal carcinoma in children. Journal of Cancer Research and Practice. 3:84-88

5. Wei KR, Zheng RS, Zhang SW, Liang ZH, Li ZM, Chen WQ (2017) Nasopharyngeal carcinoma incidence and mortality in China, 2013. Chin J Cancer. 36(1):90

6. Afqir S, Ismaili N, Alaoui K, Ahid S, Lotz JP, Horn E et al (2009) Nasopharyngeal carcinoma in adolescents: a retrospective review of 42 patients. Eur Arch Otorhinolaryngol. 266:1767-1773

7. Laskar S, Bahl G, Muckaden M, Pai SK, Gupta T, Banavali S, Arora B, Sharma D, Kurkure PA, Ramadwar M, Viswanathan S (2008) Nasopharyngeal carcinoma in children: comparison of conventional and intensity-modulated radiotherapy. Int J Radiat Oncol Biol Phys 72(3):728-736

8. Parkin DM, Bray F, Ferlay J, Pisani P (2005) Global cancer statistics 2002. CA A Cancer J Clin. 55:74-108

9. Chua ML, Wee JT, Hui EP, Chan AT (2016) Nasopharyngeal carcinoma. Lancet. 387(10022):1012-1024

10. Lo KW, To KF, Huang DP (2004) Focus on nasopharyngeal carcinoma. Cancer Cell. 5(5):423-428

11. Chong VF, Ong CK (2008) Nasopharyngeal carcinoma. Eur J Radiol. 66:437-447
12. Vokes EE, Liebowitz DN, Weichselbaum RR (1997) Nasopharyngeal carcinoma. Lancet. 350:1087-1091

13. Sultan I, Casanova M, Ferrari A, Rihani R, Rodrigueze-Galindo C (2010) Differential features of nasopharyngeal carcinoma in children and adults: a SEER study. Pediatr Blood Cancer. 55:279-284

14. Zhang L, Chen Q, Liu H, Liu L, Mai H (2013) Emerging treatment options for nasopharyngeal carcinoma. Drug Des Dev Ther 7:37-82

15. Youssef $A A$, Raafat $T A$ (2015) Nasopharyngeal carcinoma: imaging features of unusual cancer in children. The Egyptian Journal of Radiology and Nuclear Medicine. 46:943-947

16. Yi W, Liu ZG, Li X, Tang J, Jiang CB, Hu JY et al (2016) CT-diagnosed severe skull base bone destruction predicts distant bone metastasis in early Nstage nasopharyngeal carcinoma. Onco Targets Ther. 9:7011

17. Nikitovic M, Popovic-Vukovic M, Stanic D, Bokun J, Paripovic L, llic V et al (2018) Treatment outcome of childhood nasopharyngeal carcinoma: a single institution experience. International journal of pediatric otorhinolaryngology. 113:168-172

18. Eriksen JG (2018) Radiotherapy in Asia: making progress step by step. Radiotherapy and Oncology. 129(1):1-2

19. Chen WC, Jackson A, Budnick AS, Pfister DG, Kraus DH, Hunt MA, Stambuk H, Levegrun S, Wolden SL (2006) Sensorineural hearing loss in combined modality treatment of nasopharyngeal carcinoma. Cancer. 106(4):820-829

20. Ayan I, Kaytan E, Ayan N (2003) Childhood nasopharyngeal carcinoma: from biology to treatment. Lancet Oncol. 4:13-21

21. Patel PN, Penn EB (2016) Presentation and management of nasopharyngeal carcinoma, a rare childhood malignancy. Int J of Pediatric Otorhinolaryngol Extra. 14:20-22

22. Lee AW, Ma BB, Ng WT, Chan AT (2015) Management of nasopharyngeal carcinoma: current practice and future perspective. J Clin Oncol. 33(29): 3356-3364

23. Maithrea N, Periyathamby S, Mohamad I (2017) Trismus as a rare presenting symptom in a pediatric nasopharyngeal Carcinoma. Egyptian Journal of Ear, Nose, Throat and Allied Sciences. 18:91-93

24. Casanova M, Ozyar E, Patte C et al (2016) International randomized phase 2 study on the addition of docetaxel to the combination of cisplatin and 5fluorouracil in the induction treatment for nasopharyngeal carcinoma in children and adolescents. Cancer Chemother Pharmacol. 77:289-298

25. Casanova M, Bisogno G, Gandola L et al (2012) A prospective protocol for nasopharyngeal carcinoma in children and adolescents: the Italian Rare Tumors in Pediatric Age (TREP) project. Cancer. 118:2718-2725

26. Cheuk DK, Billups CA, Martin MG et al (2011) Prognostic factors and longterm outcomes of childhood nasopharyngeal carcinoma. Cancer. 117:197-206

\section{Publisher's Note}

Springer Nature remains neutral with regard to jurisdictional claims in published maps and institutional affiliations.

\section{Submit your manuscript to a SpringerOpen ${ }^{\circ}$ journal and benefit from:}

- Convenient online submission

- Rigorous peer review

- Open access: articles freely available online

- High visibility within the field

- Retaining the copyright to your article

Submit your next manuscript at $\boldsymbol{\nabla}$ springeropen.com 